\title{
Component Dependence of Aggregation Structure and Light Scattering Properties of Polymer/Liquid Crystal Composite Films
}

\author{
Kwansun Park, Hirotsugu KIKUChI, and Tisato KaJiYama* \\ Department of Chemical Science and Technology, Faculty of Engineering, \\ Kyushu University, 6-10-1 Hakozaki, Higashi-ku Fukuoka 812, Japan
}

(Received December 3, 1993)

\begin{abstract}
The aggregation structure and electro-optical properties of polymer/liquid crystal (LC) composite films were investigated. The aggregation structure of the composite film strongly depended on the components ratio of matrix polymer and low molecular weight liquid crystalline materials. The continuous LC phase was formed in a three dimensional polymer network when the $\mathrm{LC}$ weight fraction was above around $50 \mathrm{wt} \%$. Also, the aggregation structure of the composite film could be controlled by controlling the solvent evaporation velocity during the film preparation process. The finer matrix polymer fibrils were formed in the case of the faster solvent evaporation velocity. The composite films exhibited reversible light scattering-light transmission switching upon electric field -OFF and -ON states, respectively. The light scattering properties of the composite film with a continuous LC phase were strongly dependent on the spatial distortion of nematic directors as well as the mismatch in refractive indices between matrix polymer and LC upon an electric field -OFF state. By controlling the polymeric wall thickness of the composite film below the wavelength $(632.8 \mathrm{~nm})$ of an incident $\mathrm{He}-\mathrm{Ne}$ laser beam, the composite films which show a remarkably high transmittance and contrast were successfully realized.
\end{abstract}

KEY WORDS Polymer / Liquid Crystal (LC) Composite Film / Light Scattering / Components Ratio / Aggregation Structure / Light Valve Thickness of Matrix Polymer Fibril /

The polymer/liquid crystal (LC) composite films developed by Kajiyama et al. In $1979^{1}$ have continuous LC channels in a three dimensional polymer network in the case of the LC weight fraction above around $50 \mathrm{wt} \%$. Since the matrix polymer mechanically supports the LC phase and protects it from the environment, this composite film was named as the self-supported LC (SSLC) or the bicontinuous LC (BCLC) film. The SSLC composite film could be prepared by the water-casting method. ${ }^{2}$ Anisotropic electric and optical characteristics of the LC materials have been widely applied to various electro-optical systems. The most common LC display system is twisted-nematic (TN) system. ${ }^{3}$ This TN system is based on the principle that the twisted
LC alignment can rotate the plane of the polarized light by $90^{\circ}$. This system needs two polarizers that cause a low contrast and a narrow view angle. Another important invention for the LC display of nematic LC was the use of dynamic scattering mode (DSM). ${ }^{4}$ In this system, the application of a high-enough electric field induces the light scattering by the turbulence of LC which is caused by an electric current effect. LC displays with large-area, high contrast and wide-view angle have been requested for a long time. For the past around 10 years, new types of LC display devices being composed of (polymer/LC) composite films, nematic curvilinear aligned phase (NCAP), $\left.{ }^{5}\right)$ polymer dispersed LC (PDLC), ${ }^{6}$ and $\mathrm{SSLC}^{7-10}$ ) have been studied for the possible application

\footnotetext{
* To whom correspondence should be addressed.
} 
to a large-area and flexible display. The display mechanism of the composite film is based on the light scattering upon an electric field -OFF state. The light scattering of the composite film upon an electric field -OFF state is basically caused by the mismatch in refractive indices between matrix polymer and LC and also, spatial distortion of nematic directors. ${ }^{8-10}$ And the distortion of nematic directors in the LC channels is strongly dependent on the aggregation structure of the composite film. The aggregation structure of the composite film strongly depends on the components ratio ${ }^{8,11}$ ) and the solvent evaporation velocity during the film preparation process. ${ }^{12)}$ Therefore, studies on the relationships between aggregation structure and light scattering properties of the composite film are important to establish the mechanism of light scattering in the composite film.

In this paper, the component dependence of the aggregation structure and light scattering properties of the composite films and also, the origins of light scattering of the composite film have been investigated.

\section{EXPERIMENTAL}

Figure 1 shows the chemical structures and physical properties of matrix polymers and LC for the composite films. The molecular weight of the matrix polymers are around $1.5 \times 10^{5}$. The thermotropic nematic LC used in this study (E8, Merck, Ltd.) is an eutectic mixture of several cyanobiphenyl derivatives and cyanoterphenyl one with a positive dielectric anisotropy. The phase transition temperature, $T_{\mathrm{NI}}$ from nematic to isotropic state of $\mathrm{E} 8$, the phase transition temperature, $T_{\mathrm{KN}}$ from crystal to nematic state of E8 and the glass transition temperature, $T_{\mathrm{g}}$ of the matrix polymer were measured by using the differential scanning calorimetry (DSC, Rigaku DSC-8230) analysis. The scanning rate of DSC was $5 \mathrm{~K} \mathrm{~min}^{-1}$ under a nitrogen atmosphere. The composite films were prepared from a chloroform solution

\section{Polymer}

1) Poly(diisopropylfumarate) (Pdi-iPF)

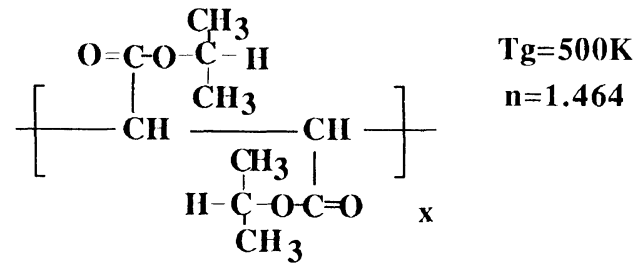

2) Poly(isopropyl-ter.-butylfumarate)(Pil-tBtF)<smiles>[Y]C([Y])(C)[C@H](C(=O)OC(C)(C)C)[C@H](C)C(=O)OC(C)(C)C</smiles>

3) Poly (diter.-butylfumarate)(PditBtF)

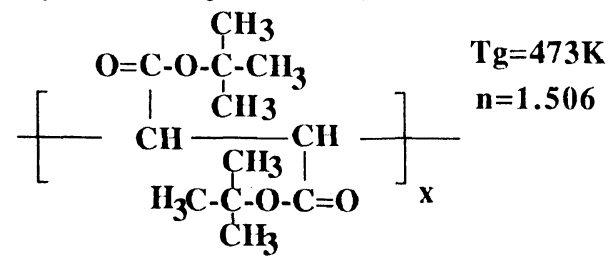

\section{Liquid crystal}

E8(Nematic mixture) (Merck LTD)

\begin{tabular}{|c|c|c|}
\hline Code & Chemical structure & Wt.(\%) \\
\hline $\mathrm{K}-15$ & $-\mathrm{C}_{5} \mathrm{H}_{11}$ & $45 \%$ \\
\hline M-9 & $\mathrm{NC}-\mathrm{O}-\mathrm{O}-\mathrm{OC}_{3} \mathrm{H}_{7}$ & $16 \%$ \\
\hline$M-15$ & (O) $-\mathrm{OC}_{5} \mathrm{H}_{11}$ & $12 \%$ \\
\hline$M-24$ & $-\left(0-\mathrm{CC}_{8} \mathrm{H}_{17}\right.$ & $16 \%$ \\
\hline$T-15$ & ○) $-\left(\mathrm{O}-\mathrm{C}_{5} \mathrm{H}_{11}\right.$ & $11 \%$ \\
\hline \multicolumn{3}{|c|}{$\begin{array}{l}\mathrm{T}_{\mathrm{KN}}=261 \mathrm{~K}, \\
\mathrm{n}_{11}=1.774 \\
\mathrm{n}_{\mathrm{AVE}}=1.609\end{array}$} \\
\hline
\end{tabular}

Figure 1. Chemical structures and physical properties of the matrix polymer and LC for the composite film.

of a mixture of polymer and LC materials by bar-coating it with a doctor blade on the indium tin oxide (ITO) coated glass at room temperature. The concentration of the solution was $10 \mathrm{wt} \%$. The weight fraction of $\mathrm{LC}$ in the composite film was changed from $0 \%$ to $95 \%$. 
The aggregation structure of the composite film was controlled by changing the velocity of solvent (chloroform) evaporation by using the experimental equipment shown in Figure 2. The solvent evaporation velocity during a film preparation process was controlled by a pump and a needle valve. The final thickness of the composite films was $8 \mu \mathrm{m}$. The aggregation structure of the composite film was investigated by scanning electron microscopic (SEM, Hitachi S-430) observation. For the SEM observation, the composite film was fractured in a liquid nitrogen before the extraction of LC molecules with methanol at room temperature.

The transmittance and electro-optical properties of the composite film were measured by means of the measuring system schematically shown in Figure 3. He-Ne laser (wavelength: $632.8 \mathrm{~nm}$ ) was used as a light source. The composite film was sandwiched between two pieces of ITO coated glasses to investigate the electro-optical properties. The intensity of transmitted light through the composite film was detected by a photodiode. The sample in Figure 3 can be heated from room temperature to $473 \mathrm{~K}$.

\section{RESULTS AND DISCUSSION}

\section{Component Ratio Dependence of the Aggrega- tion Structure and Light Scattering Properties of the Composite Films}

Figure 4 shows the SEM observation photographs of the (Pdi-iPF/E8 $=20 / 80 \mathrm{wt} \%$ ) composite film after the extraction of LC molecules. The composite film showed a unique phase separation structure in which an entirely continuous LC phase was embedded in a three dimensional polymer fibrillar network. The long range orientation of the nematic molecules in the composite film should be distorted by the randomly formed polymer fibrillar networks. Therefore, the nematic directors in the composite film are randomly distorted and discontinuous states. Because of that, the composite film (thickness, $8 \mu \mathrm{m}$ ) showed very

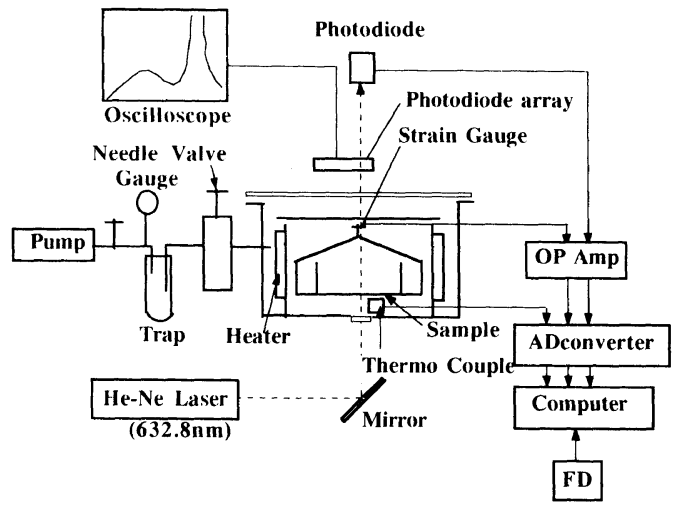

Figure 2. Measuring system of the light scattering intensity and weight change of the solution during the film formation process.

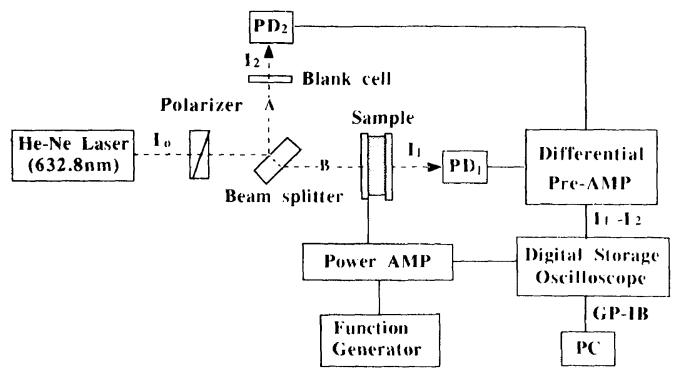

Figure 3. Schematic diagram of the measuring system for electro-optical properties.

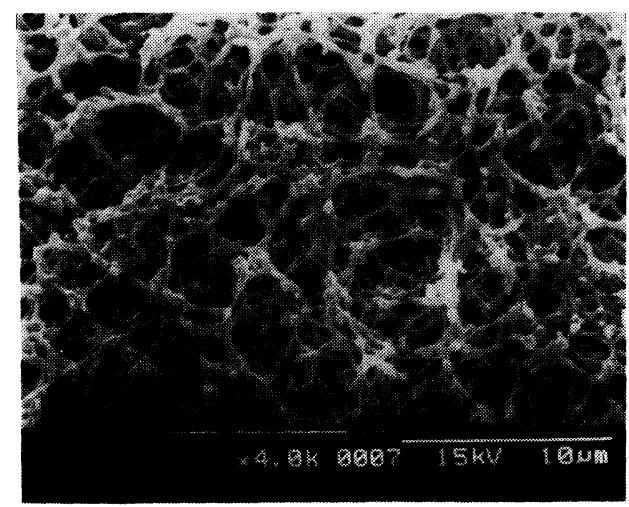

Figure 4. SEM photographs of the (Pdi-iPF/E8 $=20 / 80$ wt\%) composite film.

strong light scattering (almost $0 \%$ of transmittance). Figure 5 shows the variations of the transmittance with E8 weight fraction upon an electric field -OFF state and also, aggregation 


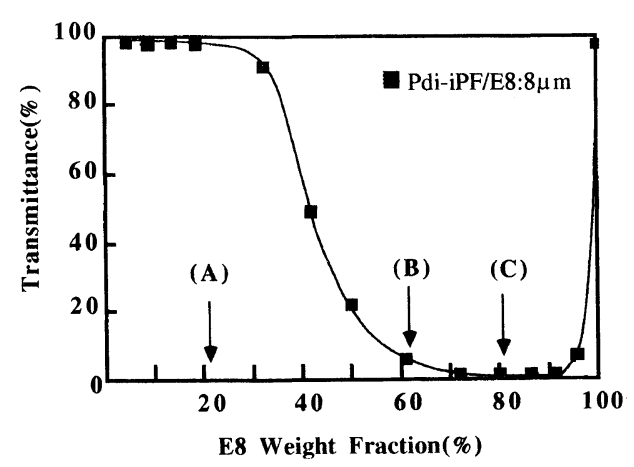

(A)

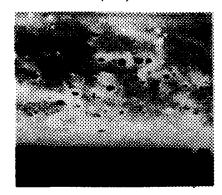

(Pdi-iPF/E8)

$(80 / 20 w t \%)$
(B)

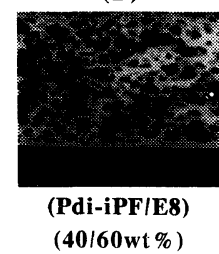

(C)

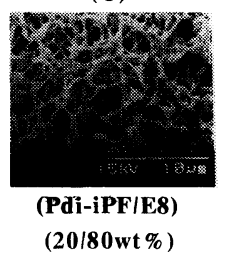

Figure 5. Variations of the transmittance with E8 weight fraction upon an electric field-OFF state and SEM photographs at points (A), (B), and (C) of the (Pdi-iPF/E8) composite film.

structures for the (Pdi-iPF/E8) composite film. The light transmittance of the composite films decreased with an increase of LC weight fraction, and then, reached to the minimum light transmittance at a point (C). In other words, the strongest light scattering appeared for the composite film (C) (polymer/LC $=20$ / $80 \mathrm{wt} \%)$. In the case of the composite film (A) (polymer/LC $=80 / 20 \mathrm{wt} \%$ ), E8 exists as isolated droplets in the matrix polymer. Therefore, the aggregation structure of the composite film (A) resembles to that of $\mathrm{NCA}^{5}$ or $\mathrm{PDLC}^{6}$ film. Then, the light scattering intensity of the composite film (A) may depend mainly on the mismatch of refractive indices between Pdi-iPF and E8 as well as the size of droplet. Since some droplets are too small to scatter the light, the light scattering intensity of the composite film (A) is not so strong. In general case, the size of spherical droplets becomes larger with an increase of E8 weight fraction. Then, the connected E8 droplets are observed with SEM when the E8 weight fraction was above $40 \%$. The abrupt decrease of transmittance for the (Pdi-iPF/E8 $=60 / 40 \mathrm{wt} \%$ ) composite film as shown in Figure 4 indicates that the formation of continuous LC channels is important for the strong light scattering. E8 in the composite film (B) $($ polymer $/ \mathrm{LC}=40 / 60 \mathrm{wt} \%)$ formed the continuous LC channels in a three dimensional polymer network. As mentioned in the previous reports, ${ }^{7-9}$ the light scattering of the composite film (B) must arise from both the optical mismatching (the difference in refractive indices between matrix polymer and LC) and the spatial distortion of nematic directors upon an electric field -OFF state. In the case of an electric field -ON state, the distortion of nematic directors almost disappears, as the long axes of LC molecules with a positive dielectric anisotropy orient along the direction of an applied electric field. Since the refractive index of LC to a normal incident light of the composite film upon an electric field - $\mathrm{ON}$ state corresponds to the ordinary refractive index of LC, $n_{0}$, the transmittance of the composite film (B) depends on the difference between refractive indices of polymer $\left(n_{\mathrm{p}}\right)$ and $n_{0}$, $\left|n_{\mathrm{p}}-n_{0}\right|$.

In the case of the composite film (C) (polymer/LC $=20 / 80 \mathrm{wt} \%$ ), the aggregation structure was similar to that of the composite film (B). However, the matrix polymer in the composite film (C) formed the bundle of thinner fibrils. Since the three dimensionally entangled and curved polymer fibrils generate a numerous number of polymeric wall to connect with LC molecules, the aggregation structure of the composite film (C) must induce the strong spatial distortion of nematic directors. And the composite films of which the LC weight fraction is around $80-90 \%$ showed polymer fibrillar network structure such as the composite film (C) and strong light scattering (low transmittance) state upon an electric field -OFF state as shown in Figure 5. If the thickness of fibrils is thinner than the wavelength ( $400-800 \mathrm{~nm})$ of an incident light, 


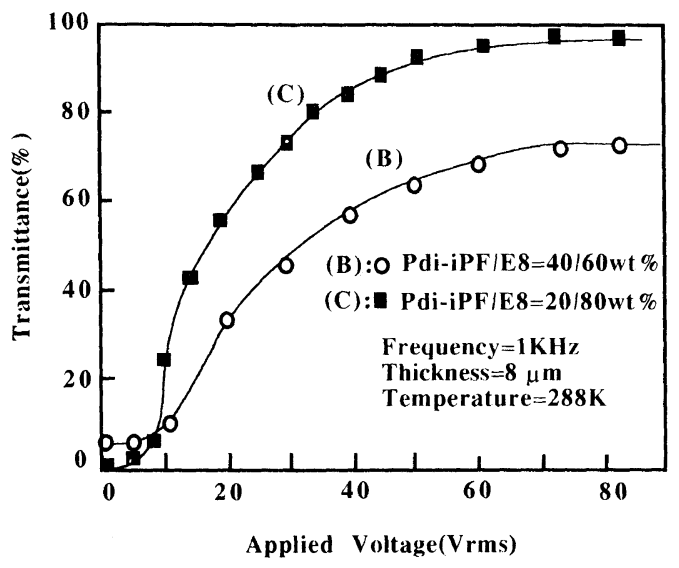

Figure 6. Variations of the transmittance of the composite films (B) and (C) in Figure 5 with an applied voltage.

it may be advantageous to obtain a high transparent state upon an electric field $-\mathrm{ON}$ state.

Figure 6 shows the variations of transmittance of the composite films (B) and (C) shown in Figure 5 with the magnitudes of an applied voltage. In spite of the lower transmittance (almost $0 \%$ ) of the film (C) than that $(7 \%)$ of the composite film (B) upon an electric field -OFF state (0 Vrms), the maximum transmittance $(93 \%)$ of the composite film (C) was higher than that $(75 \%)$ of the composite film (B) upon an electric field -ON state. The lower transmittance of the composite film (C) than that of composite film (B) upon an electric field -OFF state $(0 \mathrm{Vrms})$ came from that the composite film (C) has much more number of polymeric walls to contact with LC molecules which induce the strong spatial distortion of nematic directors as explained in Figure 5. In the case of an electric field -ON state, the nematic directors orient along the direction of an applied electric field. Since the refractive index of LC to normal incident light of the composite film upon an electric field -ON state corresponds to the ordinary refractive index, $n_{0}$, the transmittance of the composite film (B) depends on the magnitudes of $\left|n_{\mathrm{p}}-n_{0}\right|$. The magnitudes of $\left|n_{\mathrm{p}}-n_{0}\right|$ of the composite films (B) and (C) are same because the components of them are same. If the light scattering of the composite film upon an electric field -OFF state only depends on the mismatch between $n_{\mathrm{p}}$ and refractive index of $\mathrm{LC}$, the lower transmittance $(0 \%)$ of the composite film (C) than that $(7 \%)$ of the composite film (B) means that the composite film (C) has the much more light scattering points between matrix polymer and LC than those of the composite film (B), because their components, polymer and LC are same. Then, the transmittance of the composite film (C) would be lower than that of the composite film (B) upon an electric field - ON state. But the actual transmittance $(93 \%)$ of the composite film (C) was higher than that $(75 \%)$ of the composite film (B) upon an electric field -ON state as shown in Figure 6. This result clearly indicates that there exists another factor of light scattering in the composite film upon an electric field -OFF state in addition to the mismatch in refractive indices between matrix polymer and LC. We have insisted that the distortion of nematic directors in a three dimensional polymer network is one of the origins of strong light scattering of the composite film. ${ }^{7-9}$

In spite of the large mismatch in refractive indices between $n_{\mathrm{p}}$ (1.464) of Pdi-iPF and $n_{0}$ (1.527) of E8 and also, strong distortion of nematic directors being induced by many interfaces between polymeric wall and LC molecules, the composite film (C) showed the highest transmittance upon an electric field $-\mathrm{ON}$ state. This may be arisen from the fact that a considerable fraction of polymeric fibril diameter is too small in comparison with the wavelength of $\mathrm{He}-\mathrm{Ne}$ laser beam to scatter an incident $\mathrm{He}-\mathrm{Ne}$ laser beam. Therefore, it is reasonable to consider that the light scattering originated from the mismatch in refractive indices between matrix polymer and LC is not so strong upon an electric field - $\mathrm{ON}$ state in the case of the composite film (C). The light scattering state of the composite film (C) upon an electric field -OFF state is more strongly dependent on the distortion of nematic di- 


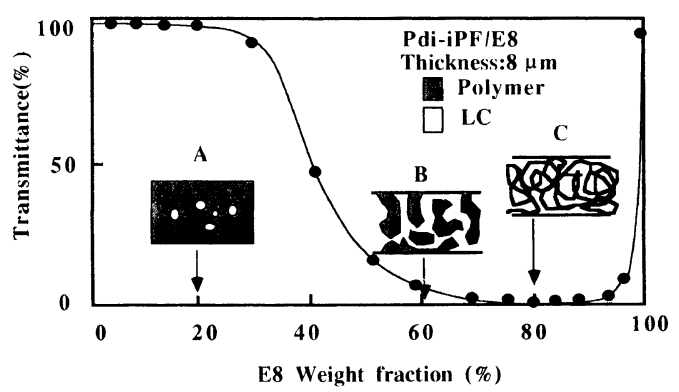

\begin{tabular}{|l|l|}
\hline Film & \multicolumn{1}{|c|}{ Light scattering origins } \\
\hline A & $\begin{array}{l}\text { Very weak light scattering by the mismatch of } \\
\text { the refractive indices between polymer and LC }\end{array}$ \\
\hline B & $\begin{array}{l}\text { Strong light scattering by the distortion of } \\
\text { nematic directors and mismatch of the refractive } \\
\text { indices between polymer and LC }\end{array}$ \\
\hline C & $\begin{array}{l}\text { The strongest light scattering largly by the } \\
\text { strong distrotion of nematic directors }\end{array}$ \\
\hline
\end{tabular}

Figure 7. Aggregation structure-light scattering property relationships of the (Pdi-iPF/E8) composite film with E8 weight fraction.

rectors rather than the mismatch in refractive indices between matrix polymer and LC.

Figure 7 shows the schematic representation on the aggregation structures and light scattering with E8 weight fraction relationships as well as light scattering characteristics of the (Pdi-iPF/E8) composite films (A), (B), and (C). As discussed above, it is reasonable to conclude that the light scattering intensity of the composite film should depend mainly on the distortion of nematic directors rather than the mismatch in refractive indices between polymer and LC for the high contrast. How to separate the magnitude of contribution from the mismatching in refractive indices and the distortion of nematic directors to the total light scattering intensity will be discussed elsewhere. ${ }^{13}$

\section{Construction of the Composite Film with High}

Transparent-Light Scattering Contrast

Based on the light scattering concepts of the composite film as shown in Figure 7 , it is possible to design an ideal aggregation structure of the composite film for high transmittance and contrast by controlling the phase separated aggregation structure of it. To achieve a high transmittance and contrast, the light scattering intensity of the composite film should depend mainly on the distortion of nematic directors rather than the mismatch in refractive indices between polymer and $\mathrm{LC}$ as described in previous paragraphs. Figure 8 shows the aggregation structures of the composite films of which matrix polymers are fumarate derivatives as shown in Figure 1 and LC weight fraction was $80 \mathrm{wt} \%$. The composite films were prepared by a solvent evaporation method under the atmosphere pressure and room temperature except the composite film (C-1). The composite film (C-1) was prepared under the vacuum condition of $2.7 \times 10^{3} \mathrm{~Pa}$ $(20 \mathrm{mmHg})$ at room temperature by using the experimental equipment in Figure 2. The thickness of matrix polymer fibrils in the composite film (C-1) was thinner than that of the composite film (C) due to the higher solvent evaporation velocity by vacuum than that of the composite film (C) during the film formation process. It was suggested from a light scattering profile during the phase separation process that the phase separation of the composite film was generated by the spinodal decomposition mechanism. ${ }^{12}$ In the process of spinodal decomposition mechanism, the periodic solute fluctuation occurs in the solution, and the solute fluctuation which has a single wavelength is developed preferentially. When the composite film is cast under the condition of an unequilibrium state at the fast solvent evaporation rate, the polymer and LC may be frozen in the infiltrated state each other. And then, the faster the solvent evaporation is, the smaller the periodicity of solute fluctuation is. Therefore, the thickness of the matrix polymer fibrils of the composite film (C-1) of which solvent evaporation was faster than that of composite film (C) were thinner than that of the composite film (C). The composite films (D) and (E) of which matrix polymers, poly(isopropylter.-butylfumarate) (PiP-tBtF) 
(C)

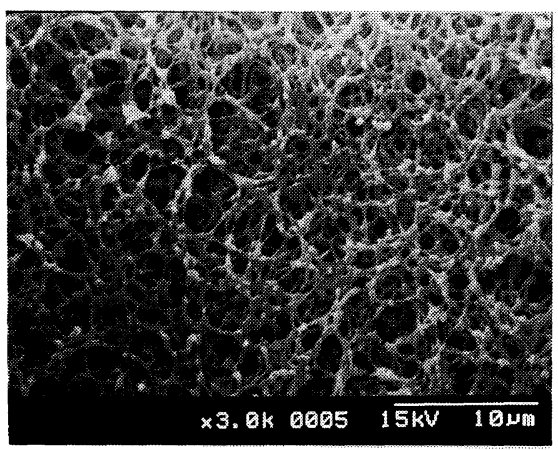

(Pdi-iPF/E8)

(D)

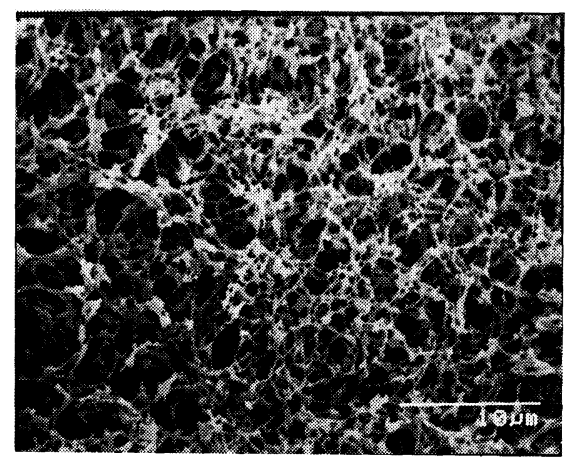

(PiP-tBtF/E8)
(C-1)

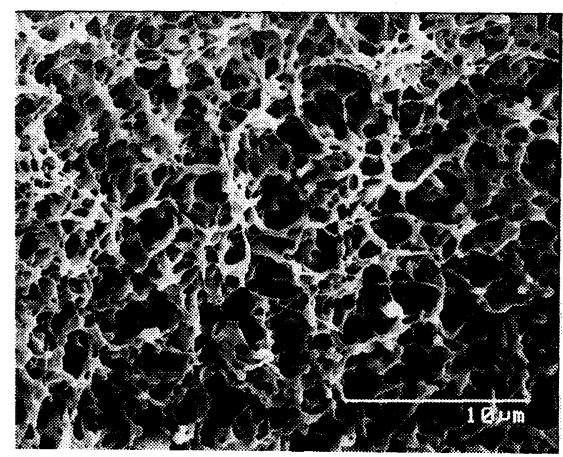

(Pdi-iPF/E8)

(E)

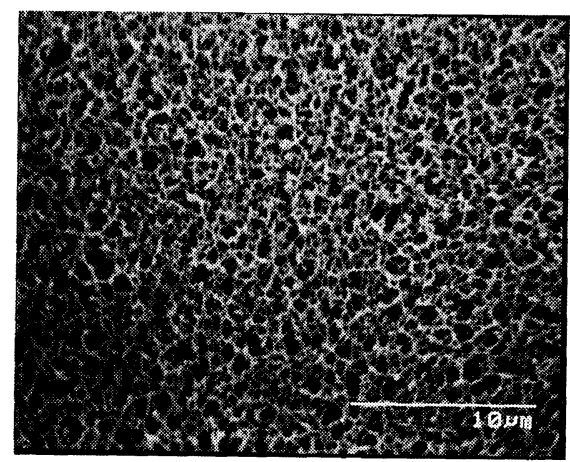

(PditBtF/E8)

\section{(Polymer/LC $=20 / 80$ wt \%) Solvent:Chloroform Temperature:298K}

Figure 8. SEM photographs of the [poly(fumarate derivatives)/E8] composite films.

and poly (diter.-butylfumarate) (PditBtF) have more bulky side chains than that of Pdi-iPF (C) as shown in Figure 1 showed the thinner matrix polymer fibrils. PiP-tBtF (D) and PditBtF (E) with more bulky side chains formed the thinner matrix polymer fibrils in the composite films. This may be due to a restricted chain diffusion during the evaporation of solvent.

Figure 9 shows the light transmittance properties of the composite films shown in Figure 8 upon electric field $-\mathrm{ON}$ and $-\mathrm{OFF}$ states. The composite film (C-1) and (C) showed same transmittance (almost $1 \%$ ) upon an electric field -OFF state. But the transmittance of the composite film (C-1) was higher than that of the composite film (C) upon an electric field -ON state in spite of the components and components ratio of them were same. Since the magnitudes of $\left|n_{\mathrm{p}}-n_{0}\right|$ and LC 


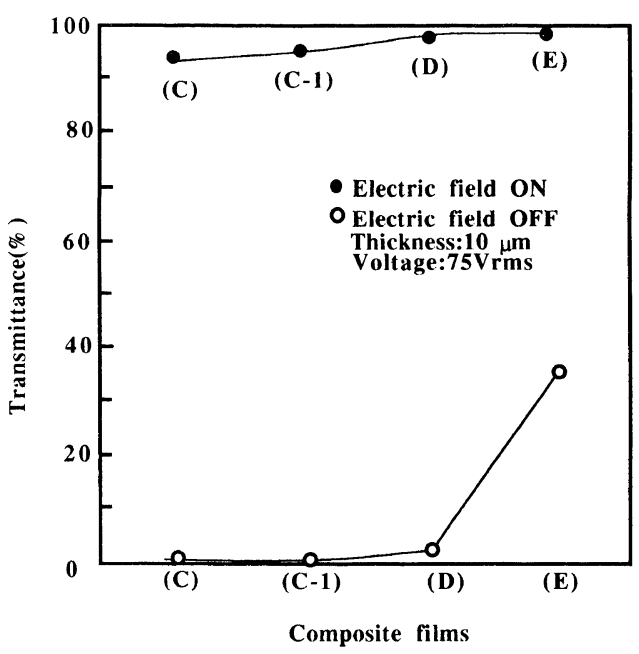

Figure 9. Transmittance of the composite film (polymer/ $\mathrm{E} 8=20 / 80 \mathrm{wt} \%$ ) of which matrix polymers are fumarate derivatives. $[(\mathrm{C}-1)$ was prepared using the equipment in Figure 2 at $\left.2.7 \times 10^{3} \mathrm{~Pa}(20 \mathrm{mmHg})\right]$.

weight fraction of composite films (C) and (C-1) are same, the difference in transmittance of the composite films (C) and (C-1) upon an electric field -ON state depends on the aggregation structure of them. The higher transmittance of the composite film (C-1) than that of the composite film (C) upon an electric field -ON state corresponds to a decrease of light scattering on the boundary of two components. Namely, the higher transmittance of the composite film (C-1) than that of the composite film (C) upon an electric field -ON state resulted from that the composite film (C-1) has thinner matrix polymer fibrils than that of the composite film (C). The light scattering is very weak when the thickness of matrix fibrils in the composite film is smaller in comparison with the wavelength of incident light and LC.

The transmittance of the composite films (D) and (E) was higher than that of the composite film (C) upon an electric field -ON state as shown in Figure 9. The considerable reasons of the higher transmittance of the composite films (D) and (E) than that of the composite film (C) upon an electric field -ON state are, 1) magnitudes of $\left|n_{\mathrm{p}}-n_{0}\right|$ for the composite films (D) (0.052) and (E) (0.021) are smaller than that of the composite film (C) (0.063), and 2) the matrix polymer fibrils in the composite films (D) and (E) are thinner than that of the composite film (C). The transmittance for the composite films (D) and (E) upon an electric field -ON state was $97-98 \%$ in spite of fairly large difference of $\left|n_{\mathrm{p}}-n_{0}\right|$ for the composite films (D) (0.052) and (E) (0.021). This result means that the light scattering on the boundary between LC and matrix polymers in the composite films (D) and (E) is so small upon an electric field -ON state. The weak light scattering on the boundary between LC and polymer in the composite films (D) and (E) in spite of the large magnitudes of $\left|n_{\mathrm{p}}-n_{0}\right|$ may be resulted from the aggregation structures of them. The thin (average, $400-500 \mathrm{~nm}$ ) matrix polymer fibrils compared with the wavelength $[632.8 \mathrm{~nm} /$ (refractive index of the composite film)] in the film might be a cause of the weak light scattering of the composite films (D) and (E). As another reason of the higher transmittance of the composite films (D) and (E) in spite of the large magnitudes of $\left|n_{\mathrm{p}}-n_{0}\right|$ upon an electric field -ON state, the decrease of the mismatches in refractive indices between LC and polymer with the change of the refractive indices of the matrix polymers by components miscibility may be considerable. But the change of the refractive indices by components miscibility in the composite films (D) and (E) could be negligible because the weight fraction of infiltrated LC molecules into the matrix polymers of the composite films (D) and (E) was below $3 \%$ against the weight of matrix polymers of them. The infiltrated LC weight fraction into the matrix polymer in the composite film was calculated by weighing the composite film and the LC extracted matrix polymer. These results mentioned above clearly indicate that fibrillar bundle of the matrix polymer should have an optimum dimension in order to obtain the high transmittance upon an electric field -ON state and the high contrast. 
The optimum dimension for the thickness of matrix polymer may be a comparable magnitude to the wavelength of an incident light.

Upon an electric field -OFF state, the transmittance of the composite film (E) showed much higher transmittance than that of the composite film (D) in spite of the almost same transmittance of them upon an electric field -ON state. From the almost same tansmittance of the composite films (D) and (E) upon an electric field -ON state, it may be true that the light scattering intensity on the boundary between LC and polymer is almost same for the composite films (D) and (E) upon an electric field -OFF state. If the light scattering intensity by the mismatch in the boundary between LC and polymer is same, the much higher transmittance of the composite film (E) than that of the composite film (D) upon an electric field -OFF state may concern with the spatial distortion of the nematic directors in the LC channels in the composite film. The dimension of the many parts of LC channels in the composite film (E) is smaller than that of the composite film (D) and the wavelength of the incident $\mathrm{He}-\mathrm{Ne}$ laser as shown in Figure 8. Therefore, these experimental results indicate that it may be possible to design an ideal aggregation structure of the composite film which shows the high transmittance and contrast.

Figure 10 shows an ideal aggregation structure of the composite film to obtain the high transmittance and contrast. The strong distortion of nematic directors may be induced due to a large number of interaction between the polymeric wall and LC molecules upon an electric field -OFF state. Therefore, the strong light scattering occurs in the composite film upon an electric field -OFF state. In the case of an electric field -ON state, the contribution of the optical mismatching to the total light scattering is reduced because of a smaller dimension of fibrillar than the wavelength of incident light. Then, the high transmittance can be retained due to disappearance of a strong

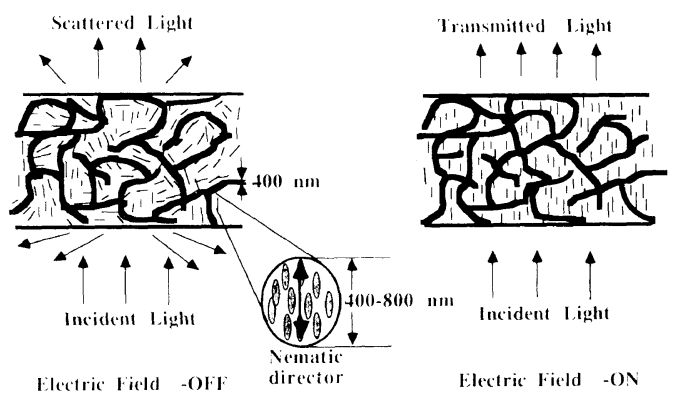

Figure 10. Ideal aggregation structure of the composite film for high contrast.

distortion of nematic directors upon an electric field -ON state.

\section{CONCLUSIONS}

The aggregation structure and light scattering properties of the composite films were investigated. The phase separated structure of the $(\mathrm{Pdi}-\mathrm{iPF} / \mathrm{E} 8=20 / 80 \mathrm{wt} \%)$ composite film showed entirely continuous LC phase in a three dimensional polymer fibrillar network. The light scattering of the SSLC composite film originated from the distortion of nematic directors as well as the mismatch in refractive indices between polymer and LC upon an electric field -OFF state. The aggregation structure and light scattering properties of the composite film strongly depended on the LC weight fraction. The strongest light scattering of the (Pdi-iPF/E8) composite film was obtained when the LC weight fraction was $80 \%$. The mismatch in refractive indices between LC and matrix polymer was not so important for the light scattering of the composite film of which matrix polymer fibrils are thinner than the wavelength of incident light. Therefore, the high transmittance and contrast composite film was possible by controlling the thickness of the matrix polymer fibrils to the smaller dimension than the wavelength of incident $\mathrm{He}-\mathrm{Ne}$ laser beam in the film (about $400 \mathrm{~nm}$ ). The aggregation structure of the composite film could be 
controlled with a different solvent evaporation speed and using the different kind of matrix polymers. The mechanism of the light scattering of the (fumarate derivative polymer/E8) composite film offered the possibility of an ideal composite film which would show high transmittance and contrast having no connection with the mismatches in refractive indices between LC and polymer.

\section{REFERENCES}

1. T. Kajiyama, Y. Nagata, E. Maemura, and Y. Takayanagi, Chem. Lett., 1979, 679.

2. T. Kajiyama, H. Kikuchi, M. Katayose, and S. Shinkai, New Polym. Mater., 1, 99 (1988).

3. M. Schadt and W. Helfrich, Appl. Phys. Lett., 18,
127 (1971).

4. R. Williams, U. S. Patent No. 3322485 (1962).

5. J. L. Fergason, SID Int. Symp. Dig. Technol., 16, 85 (1985).

6. J. W. Doane, N. V. Vaz, B. G. Wu, and S. Zumer, Appl. Phys. Lett., 48, 269 (1986).

7. T. Kajiyama, A. Miyamoto, H. Kikuchi, and Y. Morimura, Chem. Lett., 1989, 813.

8. A. Miyamoto, H. Kikuchi, Y. Morimura, and T. Kajiyama, New Polym. Mater., 2, 27 (1990).

9. A. Miyamoto, H. Kikuchi, S. Kobayashi, Y. Morimura, and T. Kajiyama, Macromolecules, 24, 3915 (1991).

10. T. Kajiyama and H. Kikuchi, J. Chem. Soc. Jpn., Chem. \& Ind. Chem., 10, 1019 (1992).

11. T. Kajiyama, H. Kikuchi, and A. Takahara, Proc. SPIE, 1665, 20 (1992).

12. T. Kajiyama, K. Park, H. Usui, H. Kikuchi, and A. Takahara, Proc. SPIE, 1911, 122 (1993).

13. Will be submitted to Polym. $J$. 